Pacific Journal of Mathematic 


\title{
THE CONSTRUCTIVITY OF MAXIMAL DUAL IDEALS IN CERTAIN BOOLEAN ALGEBRAS
}

\author{
J. C. E. DEKK ER
}

\section{Introduction}

The existence of certain mathematical entities is sometimes proved without providing any means to construct these entities. In some cases a constructive proof is found later; there are other cases where not only has no constructive proof been found, but, furthermore, it is generally suspected that no constructive proof can be found. This is especially the case for several existence theorems which are proved using the axiom of choice. For instance, all known proofs of the existence of a nonmeasurable set use the axiom of choice, and most mathematicians suspect that a constructive proof cannot be found.

There is a similar situation in Boolean algebra with regard to the existence of certain maximal dual ideals. There are two types of maximal dual ideals, the atomic ones and the nonatomic ones. While the existence of atomic maximal dual ideals can be proved without the use of the axiom of choice, all known proofs of the existence of nonatomic maximal dual ideals use the axiom of choice or an equivalent axiom. This leads to the conjecture that the atomic maximal dual ideals are in a certain sense constructive, while the nonatomic ones are not. The nature of this conjecture is, however, not clear, since no definition of a constructive dual ideal in a Boolean algebra is generally accepted.

We shall restrict our attention to two Boolean algebras whose elements are sets of nonnegative integers. Our purpose is to propose two definitions for a constructive dual ideal in these Boolean algebras based on the concept of a recursive function, and to investigate the constructivity of the maximal dual ideals in these Boolean algebras using each of these two definitions.

A collection of nonnegative integers is called a set, a collection of sets is

Received July 5, 1951, and in revised form August 29, 1952. Presented to the Society, December 27, 1950. This paper is part of a thesis submitted in partial fulfillment of the requirements for the degree of Doctor of Philosophy at Syracuse University, August 1950. The author acknowledges with gratitude the guidance and encouragement of Professor P. C. Rosenbloom during the period of preparation of his thesis.

Pacific J. Math. 3 (1953), 73-101 
called a class. Nonnegative integers and functions are denoted by small Latin letters, sets by small Greek letters, and classes by capital Latin letters. The Boolean operations are denoted by "+" for addition, "x", ".", or juxtaposition for multiplication, " " " for complementation, and " $C$ " for inclusion. Proper inclusion between classes is denoted by " $\underset{+}{C}$ ".

$$
\begin{aligned}
& \text { Nota tiONS: } \\
& V \underset{d f}{=} \text { the class of all sets, } \\
& I i(n) \underset{d f}{=} \text { the class of all sets not containing } n, \\
& L(n)=\text { the class of all sets containing } n, \\
& I \underset{d f}{=} \text { the class of all recursively enumerable (r. e.) sets, } \\
& E \underset{d f}{=} \text { the class of all recursive sets. }
\end{aligned}
$$

In the following, $B$ is a subclass of $V$ which is a Boolean algebra relative to + and $x$.

1)EFinition. The nonempty subclass $I$ of $B$ is called an ideal in $B$ if:

1) $\alpha, \beta \in I \rightarrow \alpha+\beta \in I$;

2) $\alpha \in I, \beta \in B \rightarrow \alpha \beta \in I$.

DEFINITION. The nonempty subclass $l$ of $B$ is called a dual ideal in $B$ if:

1) $\alpha, \beta \in I \rightarrow \alpha \beta \in I$;

2) $\alpha \in I, \beta \in B \rightarrow \alpha+\beta \in I$.

Definition. The ideal or dual ideal $l$ in $B$ is called proper if $l \subset B$. A MI (maximal ideal) in $B$ is a proper ideal in $B$ which is not properly included in a proper ideal in $B$. Similarly we define a MDI (maximal dual ideal) in $B$.

It is readily verified (without the use of the axiom of choice) that $W(n) \cdot B$ is a MI in $B$ and $L(n) \cdot B$ a MDI in $B$ for every value of $n$.

DEFINITION. The MI (or MDI) $M$ in $B$ is called atomic if there exists an $n$ such that $M=\mathbb{W}(n) \cdot B(\operatorname{resp} \cdot M=L(n) \cdot B)$.

The Boolean algebras which we shall consider are $[V,+, \times]$ and $[E,+, \times]$.

DEFINITION. The subclass $S$ of $F$ is recursively enumerable (r.e.) if 
either $S$ is empty, or $S$ consists only of the empty set, or there exists a recursive function $f(m, n)$ such that a nonempty set $\alpha$ belongs to $S$ if and only if there exists an $m$ such that $\alpha$ is the range of $f(m, n)$.

DEFINITION. The dual ideal $l$ in $E$ ( or $\quad$ ) is constructive in the first sense if $I\left(\right.$ resp. $\left.l \cdot F^{\prime}\right)$ is r.e.

BEFINITION. 'The dual ideal $l$ in $E$ (or $V$ ) is constructive in the second sense if there exists a r.e. subclass $S$ of $E$ (resp. $F$ ) such that $l$ consists of all sets in $E$ (resp. $V$ ) which include a set of $S$.

We now state the main results of this paper.

THEOREM A. According to each of the tuo definitions of a constructive dual ideal in $E$ or $V$ the following is true: a MDI $\|$ in $L$ or $V$ is constructive if and only if it is atomic.

THEOREM B. In the Boolean algebra E the tuo definitions are equivalent, but in the Boolean algebra $l$ constructivity in the second sense is stronger than constructivity in the first sense.

\section{Preliminaries}

1. NOTATIONS:

$0 \underset{d f}{=}$ the empty set,

$E \underset{d f}{=}$ the set of all nonnegative integers,

$\left\{n_{0}, n_{1}, \cdots, n_{k}\right\} \underset{d f}{=}$ the set consisting of $n_{0}, n_{1}, \ldots, n_{k}$,

$\left[\alpha_{0}, \alpha_{1}, \ldots, \alpha_{k}\right] \underset{d f}{=}$ the class consisting of $\alpha_{0}, \alpha_{1}, \ldots, \alpha_{k}$,

$O \underset{d f}{=}$ the empty class,

$P \underset{d f}{=}$ the class of all sets which have a finite or empty complement,

$Q \underset{d f}{=}$ the class of all sets which are finite or empty,

$R \underset{d f}{=} P+Q$

$D=E-R$. 
2. The following statements are readily verified: $P$ is a dual ideal in $V, Q$ is an ideal in $V$, and neither $P$ nor $Q$ is maximal in $V$; $R$ is a subalgebra of $V$ in which $P$ is a MDI and $Q$ a MI. If $I$ is any ideal in $V$, then $\circ \in I$, and $I$ is proper if and only if $\epsilon \notin I$. Dually, if $I$ is any dual ideal in $V$, then $\epsilon \in I$, and $I$ is proper if and only if $\circ \notin I$.

DEFINITION. The subset $K$ of the Boolean algebra $B$ is called a product system if $K$ is closed under the product operation.

One of the important theorems in Boolean algebra is: if $K$ is a product system not containing the null element, then $K$ is included in at least one MDI [4, pp. 21,22 ; this proof uses Zorn's lemma]. We shall refer to this theorem as "the theorem of the product system." It can be proved that the ideal or dual ideal $l$ in $B$ is maximal if and only if, for any $\alpha \in B, I$ contains exactly one of the two sets $\alpha$ and $\alpha^{\prime}$. The dual ideal $I$ in $B$ is therefore maximal if and only if $B-I$ (i.e., the complement of $I$ relative to $B$ ) consists exactly of the complements of the elements in $l$. Thus $I$ is a MDI in $B$ if and only if $B-I$ is a MI in $B$.

The existence of MDI's follows from the existence of MI's by the duality principle. The existence of MI's (often called prime ideals) is proved by Tarski [7], Stone [6], and Frink [1]. These proofs are existence proofs; each of them uses the axiom of choice in one of its forms.

A dual ideal $I$ in $V$ is called an extension of $P$ in $V$ if it includes $P$; $I$ is called a proper extension of $P$ in $V$ if $l \subset_{+} V$ and a maximal extension of $P$ in $V$ if it is a MDI. An ideal $I$ in $V$ is called an extension of $Q$ in $V$ if it includes $Q ; I$ is called a proper extension of $Q$ in $V$ if $I C_{+} V$, and a maximal extension of $Q$ in $V$ if it is a MI. The following theorem is well known: a MI in $V$ is nonatomic if and only if it is an extension of $Q$ in $V .{ }^{1}$ Dually: a MDI in $V$ is nonatomic if and only if it is an extension of $P$ in $V$. The expression "nonatomic MDI in $V$ "' is therefore synonymous with the expression "maximal extension of $P$ in $V$." Atomic MDI's in $V$ exist, since $L(n)$ is a MDI for every $n$; the existence of nonatomic MDI's in $V$ follows from the fact that we can apply the theorem of the product system to $P$, since $P$ is a product system not containing 0 . This proof of the existence of nonatomic MDI's in $V$ is based on the theorem of the product system, hence on Z orn's lemma. No proof of this fact is known which does not use one of the forms of the axiom of choice.

A proper extension of $P$ in $V$ cannot contain a finite set, because $P$ contains the complement of every finite set. Since every atomic MDI in $V$ contains finite sets, we see that a MI in $V$ is nonatomic if and only if it contains only infinite

${ }^{1}$ This is an immediate consequence of a theorem proved by Tarski [8, p. 57, Satz 3.6]. 
sets.

3. We suppose the reader familiar with the following concepts: an effective ( or effectively computable) function [3], a recursive function [2], a recursively enumerable (r.e) set, and a recursive set [3]. We shall assume that a function is effective if and only if it is recursive. The following six theorems can be found in Post [3, pp. 290-292]:

1) Every recursive set is r.e. 2) There exist r.e. sets which are not recursive.

$3)$ A set is recursive if and only if it and its complement are r.e. 4) The sum or product of two r.e. sets is r.e. 5) Every set which is finite or empty or whose complement is finite or empty is recursive. 6) Every infinite r.e. set has an infinite recursive subset.

The following statements follow immediately from these six theorems. An infinite r.e. set is recursive if and only if it is the range of a strictly increasing recursive function; a nonempty r.e. set is recursive if and only if it is the range of a monotone increasing recursive function; $E \subsetneq F ; E$ and $F$ are closed under addition and multiplication; $E$ is closed under complementation, but $F$ not; $R \subset E$, and, since the set of all even nonnegative integers belongs to $E-R$, we see that $R C_{+} E ; E$ is a subalgebra of $V$ but $F$ is not; $F$ is, however, a distributive lattice with a null element (namely $O$ ) and a one element (namely $\epsilon$ ); $E$ and $F$ are clearly denumerable, since they are both infinite and there are only denumerably many recursive functions. Section $\mathrm{I} .2$ remains valid if we replace " $V$ " by " $E$ " and " $L(n)$ " by " $L(n) \cdot E$ "'.

4. The definitions of a primitive recursive and of a partial recursive function can be found in Kleene [2, pp.42, 50, 51 ].

DEFINITION. Let $\pi(y)$ stand for: $y$ has the property $\pi$. Then:

$$
\mu y\{\pi(y)\}=\left\{\begin{array}{l}
\operatorname{Min} \hat{y}[\pi(y)], \text { in case }(E y)[\pi(y)], \\
\text { undefined, in case } \sim(E y)[\pi(y)] .
\end{array}\right.
$$

Kleene proved $\left[2\right.$, p. 53] that any partial recursive function $f\left(x_{1}, \cdots, x_{n}\right)$ can be expressed in normal form, $i . e .$, in the form

$$
f\left(x_{1}, \ldots, x_{n}\right)=l\left[\mu y\left\{h\left(x_{1}, \ldots, x_{n}, y\right)=0\right\}\right],
$$

where $l(x)$ and $h\left(x_{1}, \ldots, x_{n+1}\right)$ are primitive recursive. From now on we consider a partial recursive function as given if it is given in normal form. 
DEFINITION. Let

$$
f(x)=l[\mu y\{h(x, y)=0\}]
$$

where $l(x)$ and $h(x, y)$ are primitive recursive. The steps in the computation of $f(k)$ are now defined as follows:

$$
\begin{aligned}
& \text { step } 0=\text { computation of } h(k, 0) \\
& \text { step } 1=\text { computation of } h(k, 1), \\
& \vdots \quad d f \\
& \text { ad infinitum in case } \sim(E y)[h(k, y)=0] .
\end{aligned}
$$

If $(k y)[h(k, y)=0]$ and $m=\mu y\{h(k, y)=0\}$, then:

step $\mathrm{m}$ (last step) = computation of $h(k, m)$ and of $f(k)=l(m)$.

NOTATION. $f\langle k ; n\rangle=$ step $n$ in the computation of $f(k)$, provided this step exists.

DEFINITION. J et $f(x)$ be a partial recursive function defined at $x=0$, let $s_{n}$ be one less than the number of steps required for the computation of $f(n)$, in case $f(x)$ is defined for $x \leq n$. Let $\Sigma$ be the sequence

$$
f\langle 0 ; 0\rangle, \cdots, f\left\langle 0 ; s_{0}\right\rangle, f\langle 1,0\rangle, \cdots, f\left\langle 1, s_{1}\right\rangle, f\langle 2,0\rangle, \ldots
$$

where it is understood that " $f\langle r, 0\rangle, f\langle r, 1\rangle$ " is only followed by three points, in case $f(x)$ is not recursive and $r$ is the smallest value at which $f(x)$ is not definer. The sequence $\bar{\Sigma}$ is defined in terms of $\Sigma$ as follows:

$$
\begin{aligned}
& \text { replace " } f\langle 0 ; 0\rangle,, \cdots, \text { “ } f\left\langle 1, s_{1}-1\right\rangle \text { " by } f(0) \text {, } \\
& \text { replace " } f\left\langle 1 ; s_{1}\right\rangle, \text {, } \ldots, \text { " } f\left\langle 2, s_{2}-1\right\rangle \text { ", by } f(1) \text {, } \\
& \text { replace " } f\left\langle 2 ; s_{2}\right\rangle, \text {, } \ldots, \text { " } f\left\langle 3, s_{3}-1\right\rangle \text { " by } f(2) \text {, etc. }
\end{aligned}
$$

Then $\bar{f}(n)=$ the $(n+1)^{\text {st }}$ element of $\bar{\Sigma}$.

REMARK. The bar operation maps every partial recursive function uhich is defined at $x=0$ on a recursive function in such a way that the range of $\bar{f}(x)$ equals the range of $f(x)$, in case $f(x)$ is recursive, while the range of $\bar{f}(x)$ is finite, in case $f(x)$ is not recursive.

DFFINITION. Let $f(x)$ be a recursive function. Then

$$
f^{*}(0) \underset{d f}{=} f(0), f^{*}(n+1)=\left\{\begin{array}{l}
f^{*}(n) \text { if } f(n+1)<f^{*}(n), \\
f(n+1) \text { if } f(n+1) \geq f^{*}(n) .
\end{array}\right.
$$


REMARK. The star operation maps every recursive function on a monotone increasing recursive function and every monotone increasing recursive function on itself.

T 1.1. 1) There exists a recursive function $f_{n}(x)$ of $n$ and $x$ such that every nonempty, r.e. set is the range of at least one recursive function occurring in $\left\{f_{n}(x)\right\}$.

2) There exists a recursive function $e_{n}(x)$ of $n$ and $x$ which is monotone increasing for every $n$, such that every nonempty, recursive set is the range of at least one recursive function occurring in $\left\{e_{n}(x)\right\}$.

Proof. 1) kleene proved [2, p.58] the existence of a partial recursive function of two variables, say $g_{n}(x)$, such that every partial recursive function of one variable occurs at least once in $\left\{g_{n}(x)\right\}$. There clearly exists a recursive function $a(n)$ such that $\left\{g_{a(n)}(x)\right\}$ contains all functions in $\left\{g_{n}(x)\right\}$ which are defined at $x=0$. Let

$$
f_{n}(x)=\bar{g}_{a(n)}(x)
$$

then $f_{n}(x)$ satisfies the requirements.

2) Let

$$
e_{n}(x) \underset{d f}{=} f_{n}^{*}(x)
$$

where $f_{n}(x)$ is the function described in part 1$)$; then $\left\{e_{n}(x)\right\}$ is a sequence of monotone increasing recursive functions. If $\eta$ is a nonempty recursive set there exists a monotone increasing recursive function, say $e(x)$, ranging over $\eta$; it follows that $\bar{e}(x)$ occurs in $\left\{f_{n}(x)\right\}$, hence $\bar{e}^{*}(x)$, i.e. $\bar{e}(x)$, occurs in $\left\{e_{n}(x)\right\}$. Since the range of $\bar{e}(x)$ is $\eta, e_{n}(x)$ satisfies the requirements.

5. The diagonal procedure used in the proof of the following theorem is our main tool in the investigation of the constructivity of MDI's in $E$ and $V$.

$\mathrm{T}$ 1.2. Let $S$ be a nonempty, finite or denumerable class of infinite sets. Then there exist nondenumerably many sets $\gamma$ such that both $\gamma$ and $\gamma^{\prime}$ are infinite and neither $\gamma$ nor $\gamma^{\prime}$ includes any set of $S$.

Proof. We order the sets of $S$ in an infinite sequence $\alpha_{0}, \alpha_{1}, \ldots$. If $S$ is finite this can be done by repeating one of its sets infinitely many times in the sequence. Let $a_{k 0}, a_{k_{1}}, \ldots$ be an enumeration without repetitions of $\alpha_{k}$; this 
sequence is called row $k$ for purposes of presentation we shall call the set $\gamma$ a $\beta$-set if both $\gamma$ and $\gamma^{\prime}$ are infinite, while neither $\gamma$ nor $\gamma^{\prime}$ includes any set of $S$. Let

$$
\begin{aligned}
& c_{0}=a_{00}, \\
& d f=a_{01}, \\
& d_{1}=a \\
& c_{1}=\text { the first element in row } 1 \text { which is }>\operatorname{Max}\left(c_{0}, d_{0}\right), \\
& d_{1}=\text { the second such element in row } 1, \\
& c_{2}=\text { the first element in row } 2 \text { which is }>\operatorname{Max}\left(c_{0}, d_{0}, c_{1}, d_{1}\right), \\
& d_{2}=\text { the second such element in row } 2 \text {, etc. }
\end{aligned}
$$

Suppose

$$
\gamma \underset{d f}{=}\left\{c_{0}, c_{1}, \cdots\right\}, \delta \underset{d f}{=}\left\{d_{0}, d_{1}, \cdots\right\}
$$

then we observe: both $\gamma$ and $\delta$ are infinite, $\gamma$ and $\delta$ are disjoint, both $\gamma$ and $\delta$ have at least one element in common with every row. Thus $\gamma^{\prime}$ is infinite, since $\gamma^{\prime} \supset \delta$ and $\delta$ infinite; moreover each of the two statements $\gamma \supset \alpha_{n}$, $\gamma^{\prime} \supset \alpha_{n}$ is false for every $n$. We conclude that $\gamma$ is a $\beta$-set. If $\gamma_{0}, \cdots, \gamma_{k}$ are $\beta$-sets we can prove the existence of a $\beta$-set $\gamma_{k+1}$ different from $\gamma_{i}$ for $i=$ $0, \cdots, k$ by applying the same diagonal procedure to $\gamma_{0}, \ldots, \gamma_{k}, \alpha_{0}, \alpha_{1}, \ldots$ It follows that the number of $\beta$-sets is not finite. If there were only denumerably many $\beta$-sets, they could be ordered in a sequence, say $\left\{\delta_{n}\right\}$; by applying our diagonal procedure to $\alpha_{0}, \delta_{0}, \alpha_{1}, \delta_{1}, \ldots$ we would obtain a $\beta$-set $\delta$ such that $\delta \neq \delta_{n}$ for every $n$. The number of $\beta$-sets is therefore nondenumerable.

6. The following theorem, which deals with the relation between dual ideals in $V$ and dual ideals in $E$, can easily be verified by the reader.

T 1.3. 1) Any dual ideal in $V$ intersects $E$ in a dual ideal in $E$.

2) Any MDI in $V$ intersects $E$ in a MDI in $E$.

$3)$ If $M$ is a MDI in $V$, then the MDI $M \cdot E$ in $E$ is atomic if and only if $M$ is atomic.

7. SUMmARY. Though our paper is primarily concerned with the constructivity of dual ideals in $E$ and $V$, we have included some theorems about dual 
ideals in $E$ and $V$ which may be interesting for their own sake. In $\S$ II we shall discuss whether several important subclasses of $F$ are r.e. and prove Theorem A in so far as it deals with constructivity in the first sense. An extension $I$ of $P$ in $E($ or $V$ ) is called simple if there exists a set $\alpha$ in $E$ (resp. in $V$ ) such that $I$ is the intersection of all extensions of $P$ in $E$ (resp. in $V$ ) which contain $\alpha$. In $\{$ III simple extensions of $P$ in $E$ and $V$ are studied, and a second proof is given of the fact that a nonatomic MDI in $E$ ( or $V$ ) is not constructive in the first sense. An extension of $P$ in $L($ or $V$ ) is called semisimple if it can be expressed as a finite or denumerable sum of simple extensions of $P$ in $E$ (resp. $V)$. In $\$$ IV semisimple extensions of $P$ in $E$ and $V$ are discussed, Theorem $A$ is proved in so far as it deals with constructivity in the second sense, and the relation between the two types of constructivity is investigated (Theorem B).

\section{Recursively enumerable classes}

1. Both the following definitions for a "constructive set" seem reasonable:

1) "The set $\alpha$ is constructive, if there exists an effective method which enables us to decide in a finite number of steps for any given nonnegative integer $n$ whether or not $n$ belongs to $\alpha_{.}$"' This is in the spirit of Kronecker who required of a definition that it should include an effective criterion which permits us to determine for any given object whether or not it satisfies the conditions specified in the definition.

2) "The set $\alpha$ is constructive if $\alpha$ is either empty, or finite, or an infinite set which can be effectively generated in a sequence $\left\{a_{n}\right\}$ of different elements."

The first definition amounts to " $\alpha$ is constructive if $\alpha$ is recursive" and the second one to " $\alpha$ is constructive if $\alpha$ is r.e." Since $E \subset F$, we see that the second definition is weaker than the first. If we use either " $\alpha \in E$ " or " $\alpha \in F$ " as a definition for " $\alpha$ is constructive," it seems natural to define the constructivity of a (possibly nondenumerable) class $S$ of sets by some suitable property of $S \cdot E$ (resp. $S \cdot F)$. In this way we are led to the problem of finding properties of a constructive character for subclasses of $E$ and subclasses of $F$.

2. DEFINITION. The characteristic function $\operatorname{char}_{\alpha}(n)$ of a sei $\alpha$ is defined by

$$
\operatorname{char}_{\alpha}(n)=\left\{\begin{array}{l}
1 \text { for } n \in \alpha, \\
0 \text { for } n \notin \alpha .
\end{array}\right.
$$


DEFINITION. The class $K$ is called characteristically recursively enumerable (ch.r.e.) if it is a subclass of $E$ which either is empty or has the property that there exists a recursive function $k(m, n)$ such that the set $\alpha$ belongs to $K$ if and only if there exists an $m$ such that $\operatorname{char}_{\alpha}(n)=k(m, n)$.

Notation. $K=$ Char $k(m, n)$.

For the definition of a r.e. subclass of $F$ we refer to the introduction. If o $\Varangle S$ and $f(m, n)$ is related to $S$ as described in this definition, we say that $f(m, n)$ recursively enumerates $S$.

Notation. $S=$ Enum $f(m, n)$.

DEFINITION. $S^{\prime}$ is the class over which $\alpha^{\prime}$ ranges if $\alpha$ ranges over $S$.

DEFINITION. The sequence $\left\{S_{l}\right\}$ of nonempty r.e. classes which do not contain $O$ is called r.e., if there exists a recursive function $f(l, m, n)$ such that

$$
S_{l}=\operatorname{Enum} f(l, m, n)
$$

for every $l$.

The following theorem can easily be verified.

T 2.1. 1) The sum of a finite number of ch.r.e. classes is ch.r.e.

2) The subclass $K$ of $E$ is ch.r.e. if and only if $K^{\prime}$ is ch.r.e.

3 ) Every ch.r.e. class is r.e.

4) The sum of a finite number of r.e. classes is r.e.

5 ) The sum of a r.e. sequence of r.e. classes is r.e.

3. T 2.2. 1) The classes $P$ and $Q$ are both ch.r.e. and r.e.

2) The class $F$ is r.e.

$3)$ The class $E$ is r.e., but not ch.r.e.

Proof. 1) We shall first prove that $Q$ is ch.r.e. Let $n$ be any nonnegative integer, and suppose $s_{n 0}, s_{n_{1}}, \ldots, s_{n r_{n}}$ is the finite sequence of zeros and ones such that

$$
n=s_{n 0} \cdot 2^{0}+s_{n 1} \cdot 2^{1}+\cdots+s_{n r_{n}} \cdot 2^{r_{n}}
$$

This means that $n$ is written as $s_{n r_{n}} s_{n r_{n-1}} \cdots s_{n 0}$ in the dual number system. 
Observe that $n$ uniquely determines this finite sequence, and that $s_{n r_{n}}=0$ for $n=0$, while $s_{n r_{n}}=1$ for $n>0$. Let

$$
\Phi_{k}=\text { the infinite sequence } s_{k_{0}} s_{k_{1}} \cdots s_{k r_{k}} 000 \cdots
$$

and suppose $q(k, n)$ is the $(n+1)^{\text {st }}$ element in $\Phi_{k} ; q(k, n)$ is clearly a recursive function, and $\zeta=$ Char $q(k, n)$. It follows that $Q$ is ch.r.e. Then $P$ is ch.r.e., since $P=Q^{\prime}$. It is easy to see that

$$
\left(j-[0]=\operatorname{Char} q^{*}(k, n),\right.
$$

where

$$
q^{*}(k, n) \underset{d f}{=} q(k+1, n)
$$

thus $Q-[0]$ is ch.r.e., and both $Q-[0]$ and $Q$ are r.e. The class $P$ is r.e., because it is ch.r.e.

2) Let $f_{n}(x)$ be the recursive function mentioned in T 1.1.1. Then

$$
F-[\circ]=\text { Enum } f_{n}(x)
$$

and both $F-[0]$ and $F$ are r.e.

3 ) Let $e_{n}(x)$ be the recursive function mentioned in T 1.1.2. Then

$$
E-[0]=\text { Enum } e_{n}(x) \text {, }
$$

and both $E-[0]$ and $E$ are r.e. To prove that $E$ is not ch.r.e., suppose $c(m, n)$ is a recursive function such that $E=$ Char $c(m, n)$, and suppose $\gamma_{m}$ is the set with $c(m, n)$ as its characteristic function. Let

$$
d(n)=\left\{\begin{array}{l}
0 \text { for } c(n, n)=1, \\
1 \text { for } c(n, n)=0,
\end{array}\right.
$$

and let $\delta$ be the set with $d(n)$ as its characteristic function. Then $\delta \in E$, since $d(n)$ is recursive and $\delta \neq \gamma_{m}$ for every $m$, because $m \in \gamma_{m}{ }^{\prime} \delta+\gamma_{m} \delta^{\prime}$. The assumption that $E$ is ch.r.e. leads therefore, to a contradiction.

T 2.3. Let $S$ be a nonempty, finite or denumerable, r.e. subclass of $F-Q$. If a recursive function $s(m, n)$ is given which enumerates $S$, we can construct a set $\eta \in D$ such that neither $\eta$ nor $\eta^{\prime}$ includes any set of $S$. 
Proof. If $\sigma_{m}$ is the range of $s(m, n), S$ consists exactly of all sets occurring at least once in the sequence $\sigma_{0}, \sigma_{1}, \ldots$. For every $m$ we can effectively find a recursive enumeration without repetitions of $\sigma_{m}$. The diagonal procedure used in the proof of $\mathrm{T} 1.2$ becomes effective when applied to the sequence $\sigma_{0}, \sigma_{1}, \ldots$, since $s(m, n)$ is recursive.

COROLLARY 1. If $D \subset S \subset F-Q, S$ is not r.e.

Corollary 2. The classes $D, D+P, F-R, F-($ are not r.e.

4. Consider the following question: "If $M$ is a MUI in $E$ ( or $V$ ), what do we know about: 1) the characteristic recursive enumerability of $U$ (resp. $M \cdot E)$ ? $2)$ the recursive enumerability of $M(\operatorname{resp} . M \cdot F)$ ?',

T 2.4. If $M$ is a MDI in $E($ or $V)$, then $M($ resp. $M \cdot E)$ is not ch.r.e.

Proof. Note that every MDI in $V$ intersects $E$ in a MDI in $E$; it therefore suffices to prove that a MDI in $E$ is not ch.r.e. Let $M$ be a MDI in $E$. Suppose $M$ were ch.r.e.; then $M^{\prime}=E-M$ would also be ch.r.e., by $\mathrm{T} 2.1 .2$. This would imply that $E=M+(E-M)$ is ch.r.e., by $\mathrm{T} 2.1 .1$; this is, however, false by T 2.2.3. Thus $M$ is not ch.r.e.

T 2.5 If $M$ is any MDI in $E\left(\right.$ or $\left.V^{\prime}\right)$ then:

a) if $M$ is atomic, then $M(\operatorname{resp} . M \cdot F)$ is r.e.;

b) if $M$ is nonatomic, then $M($ resp. $M \cdot F)$ is not r.e.

Proof. a) I.et $M$ be an atomic MIII in $E$, say $M=L(k) \cdot E$. Suppose $e(m, n)$ is a recursive enumeration of $E-[0]$. We define

$$
a(m, n)=\left\{\begin{array}{l}
e(m, n-1) \text { for } n>0, \\
k \text { for } n=0
\end{array}\right.
$$

It is now easy to verify that $a(m, n)$ is a recursive enumeration of $L(k) \cdot E$. Using a recursive enumeration of $F-[0]$, we can similarly prove that an atomic MIDI in $V$ intersects $F$ in a r.e. class.

b) Let $M$ be a nonatomic MDI in $V$. This implies that $M \subset V-Q$ as we observed at the end of $\delta$ I.2. Thus $M \cdot F \subset F-Q$. Clearly $M \cdot F$ is nonempty. If $M \cdot F$ were r.e. there would by T 2.3 exist a set $\eta \in D$ such that $\eta, \eta^{\prime} \notin M \cdot F$; this would imply that $\eta, \eta^{\prime} \notin M$, since $\eta, \eta^{\prime} \in F$. This is, however, impossible because a MDI contains exactly one of any two complementary sets. It follows 
that $M \cdot F$ is not r.e. If $M$ is a nonatomic $M D I$ in $E$ we know $M=M \cdot E \subset E-Q \subset$ $F-Q$, and we can give a similar proof of the fact that $M$ is not r.e.

Let $l$ be a dual ideal in $E$ (or $V$ ). Then we see from T 2.4 and $\mathrm{T} 2.5$ that the definition " $I$ is constructive if $I$ (resp. $I . E$ ) is ch.r.e." is less satisfactory than " $I$ is constructive if $I$ (resp. $l \cdot F$ ) is r.e." For any MDI in $E$ or $V$ (atomic or nonatomic) would be nonconstructive according to the former definition, while according to the latter definition a MDI would be constructive if and only if it is atomic. A dual ideal in $E$ ( or $V$ ) is called constructive in the first sense if $I$ (resp. $l \cdot F$ ) is r.e. Theorem $\mathrm{T} 2.5$ is therefore identical to the part of Theorem A which deals with constructivity in the first sense.

\section{Simple extensions of $\mathbf{P}$}

1. The only proper extensions of $P$ in $V$, of which we have discussed whether they intersect $F$ in a r.e. class, are $P$ itself and maximal extensions of $P$ in $V ; P$ intersects $F$ in a r.e. class, namely in $P$ itself, and any maximal extension of $P$ in $V$ intersects $F$ in a class which is not r.e. A solution of the problem: "Which extensions of $P$ in $V$ intersect $F$ in a r.e. class?" might increase our understanding of maximal extensions of $P$ in $V$. If it would turn out that no proper extension of $P$ in $V$ which properly includes $P$ intersects $F$ in a r.e. class, the fact that maximal extensions of $P$ in $V$ do not intersect $F$ in a r.e. class would not reveal much about their nature. If the collection of proper extensions of $P$ in $V$ which properly include $P$, would, however, consist of two nonempty subcollections: those which do intersect $F$ in a r.e. class and those which do not, we might get a better insight into the nonconstructive character of maximal extensions of $P$ in $V$ by studying these two subcollections. The $\S \S$ III and IV are an attempt to determine which proper extensions of $P$ in $V$ properly including $P$ intersect $F$ in a r.e. class. The analogous problem for extensions of $P$ in $E$ is also considered.

2. Definition. $\alpha \equiv \beta=\left(\alpha \beta^{\prime}+\alpha^{\prime} \beta \in Q\right)$, i.e., $\alpha \equiv \beta$ if $\alpha$ and $\beta$ differ by at most a finite number of elements. We shall read this: “ $\alpha$ and $\beta$ are congruent.",

DEFINITION. $\left\langle\alpha_{\diamond}\right\rangle$ is the class of all sets congruent to $\alpha_{\bullet}$

$$
\begin{aligned}
\text { Definition. } & \langle\alpha\rangle+\langle\beta\rangle=\langle\alpha+\beta\rangle,\langle\alpha\rangle \times\langle\beta\rangle=\langle\alpha, \beta\rangle, \\
\langle\alpha\rangle^{\prime} & =\left\langle\alpha_{d f}^{\prime}\right\rangle .
\end{aligned}
$$

It is easy to see that these definitions are unique and that the equivalence 
classes defined by the congruence relation in $V$ form a Boolean algebra with respect to,$+ \times, '$, which has $Q$ as its null element and $P$ as its one element.

$$
\text { DEFINITION. } \nu(n) \underset{d f}{=}\{0,1, \cdots, n\} \text {. }
$$

DEFINITION. $\beta$ almost includes $\alpha$ ( or $\alpha$ is almost included in $\beta$ ), abbreviated $\beta$ alin $\alpha$, if $\alpha \beta^{\prime} \in Q$, i.e., if $\beta$ contains all but at most a finite number of elements of $\alpha^{2}$

DEFINITION. Alin $S$ is the class of all sets which almost include a set of $S$.

Obviously, $\beta$ alin $\alpha \leftrightarrow \alpha+\beta \equiv \beta \leftrightarrow$ there exists an $n$ such that $\beta \supset \alpha-\nu(n)$ $\leftrightarrow$ there exists a set $\delta \in Q$ such that $\delta \subset \alpha$ and $\beta \supset \alpha-\delta$. Also,

$$
a \equiv \beta \leftrightarrow\left\{a_{\text {alin }} \beta \text { and } \beta \text { alin } \alpha\right\}
$$

The alin relation is clearly a generalization of the inclusion relation. It is reflexive and transitive; it is, however, not a partial ordering relation, since it is not antisymmetric. For if

$$
\alpha=\{0,2,3,4, \ldots\} \text { and } \beta=\{1,2,3,4, \ldots\}
$$

then $\alpha$ alin $\beta$ and $\beta$ alin $\alpha$, while $\alpha \neq \beta$. If $\beta_{1}$ alin $\alpha_{1}$ and $\beta_{2}$ alin $\alpha_{2}$, then $\beta_{1}+\beta_{2}$ alin $\alpha_{1}+\alpha_{2}$ and $\beta_{1} \beta_{2}$ alin $\alpha_{1} \alpha_{2}$. Also $\beta$ alin $\alpha$ if and only if $\alpha^{\prime}$ alin $\beta^{\prime}$. Any set $\beta$ almost includes any finite or empty set; a finite or empty set $\beta$ almost includes a set $\alpha$ if and only if $\alpha$ is finite or empty. Alin $S=V$ whenever $S$ contains a finite set, and $A$ lin $I=I$ for any extension $I$ of $P$ in $V$. If $S$ consists of a finite number of sets, say $\alpha_{0}, \cdots, \alpha_{n}$, we shall denote Alin $S$ by $\operatorname{Alin}\left(\alpha_{0}, \cdots, \alpha_{n}\right)$

3. Let $I$ be a dual ideal in the Boolean algebra $B$. Any dual ideal in $B$ which includes $I$ is called an extension of $I$ in $B$. If $S \subset B$, we shall denote the intersection of all extensions of $I$ in $B$ which include $S$ by $I_{B}(S)$. In case $S$ consists of a finite number of elements, say $\alpha_{0}, \ldots, \alpha_{n}$, we shall denote $l_{B}(S)$ by $I_{B}\left(\alpha_{0}, \cdots, \alpha_{n}\right) . I_{B}(S)$ is obviously the smallest extension of $I$ in $B$ which includes $S$. Also

$$
I_{B}(\alpha)=[\rho \alpha+\gamma]
$$

where $\rho$ ranges over $I$ and $\gamma$ over $B$. 
Definition. The extension $I_{1}$ of the dual ideal $I$ in $B$ is called a simple extension of $l$ in $B$, if there exists an element $\alpha \in B$ such that $l_{1}=l_{B}(\alpha)$.

Evidently $P_{V}(\alpha)=$ Alin $\alpha$ for any $\alpha \in V$, and $P_{E}(a)=E$. Alin $\alpha$ for any $\alpha \in E$. Let $l$ be a simple extension of $P$ in $V$; every set $\alpha \in l$ such that $I=P_{V}(\alpha)$ is called a generator of $I$. A necessary and sufficient condition that $\alpha$ and $\beta$ are generators of the same simple extension of $P$ in $l$ is $\alpha \equiv \beta$. This means that a simple extension of $P$ in $V$ uniquely determines its generator modulo $Q$. The following statements are easily verified: $P_{V}(\alpha)=P$ if and only if $\alpha \in P ; P_{V}(\alpha)=V$ if and only if $\alpha \in Q ; P \subset_{+} P_{V}(\alpha) \subsetneq_{+} V$ if and only if $\alpha \notin R$.

T 3.1. 1) Every proper extension of $P$ in $V$ is included in at least one maximal extension of $P$ in $V$.

2) If $\beta$ does not almost include $\alpha$, there exists a simple extension $I$ of $P$ in $V$ such that $\alpha \in I, \beta \notin I$, and a maximal extension $M$ of $P$ in $V$ such that $\alpha \in M, \beta \notin M .^{3}$

3) If $\alpha$ alin $\beta$, then $P_{V}(\alpha) \subset P_{V}(\beta)$; if $\alpha$ alin $\beta$ is true, but $\beta$ alin $\alpha$ false, then $P_{V}(\alpha) \subset_{+} P_{V}(\beta)$.

4) A simple extension of $P$ in $V$ cannot be a maximal extension of $P$ in $V$.

Proof. 1 ) Every proper extension of $P$ in $V$ is a product system not containing $O$; we can therefore apply the theorem of the product system.

2) $\alpha \beta^{\prime} \notin Q$, since $\beta$ alin $\alpha$ is false. Let $I=P_{V}\left(\alpha \beta^{\prime}\right)$; then $I \subset V$; furthermore $\alpha, \beta^{\prime} \in I$, because $\alpha \beta^{\prime} \in I$. It follows that $\beta \notin I$, since $I_{+}^{+} V$ and $\beta^{\prime} \in I$. Thus $I$ is a simple extension of $P$ in $V$ satisfying the requirements. In view of 1$), l$ is included in a maximal extension of $P$ in $V$, say $M$. Consequently $\alpha, \beta^{\prime} \in M$, hence $\beta \notin M$.

3 ) Any set which almost includes a set of $I$ belongs itself to $I$, since indeed Alin $I=I$. If $\alpha$ alin $\beta$ then $\alpha \in P_{V}(\beta)$, hence

$$
P_{V}(\alpha)=\operatorname{Alin} \alpha \subset P_{V}(\beta) \text {. }
$$

Now $P_{V}(\alpha)=P_{V}(\beta)$ would imply $\alpha \equiv \beta$, hence $\beta$ alin $\alpha$. It follows that $P_{V}(\alpha) \underset{+}{\subsetneq} P_{V}(\beta)$, whenever $\alpha$ alin $\beta$ is true and $\beta$ alin $\alpha$ false.

3The second part of $\mathrm{T} 3.12$ was suggested by a result of Stone $[6, \mathrm{p} .105$, Theorem 64]. 
4) Let $I=P_{V}\left(a^{\prime}\right)$; we may assume $\alpha \notin R$, the theorem being trivial in case $\alpha \in R$. We observe that there exists an infinite set $\beta$ such that $\beta \subset \alpha$ and $\alpha-\beta \notin Q$, because $\alpha$ is infinite. But this implies that $\alpha$ alin $\beta$ is true and $\beta$ alin $\alpha$ false. Moreover, $P_{V}(\beta) \subsetneq_{+} V$, since $\beta \notin Q$. Thus $P_{V}(\alpha) \subsetneq_{+} P_{V}(\beta) \subsetneq_{+} V$; this implies that $P_{V}(\alpha)$ is not a maximal extension of $P$ in $V$.

REMARK. All four parts of this theorem remain valid, if we replace " $V$ ", by " $E$ " and assume $\alpha, \beta \in E$. In the proof of the third part we have to take a recursive subset of $\alpha$ for $\beta$. If $a(n)$ is a strictly increasing recursive function ranging over $\alpha$, we can take $\beta$ as the range of $a(2 n)$.

4. Let $I$ be a dual ideal in $B$, and suppose $\alpha_{0}, \alpha_{1}, \ldots, \alpha_{n} \in B$. It is easy to verify that

$$
I_{B}\left(\alpha_{0}, \alpha_{1}\right)=I_{B}\left(\alpha_{0} \cdot \alpha_{1}\right) \text { and } I_{B}\left(\alpha_{0}, \alpha_{1}, \ldots, \alpha_{n}\right)=I_{B}\left(\alpha_{0} \cdot \alpha_{1} \cdots \alpha_{n}\right)
$$

This means that adjunction of any finite number of elements of $B$ to $l$ leads to the same result as adjunction of a certain single element of $B$ to $I$. Clearly

$$
P_{V}(\alpha, \beta)=P_{V}(\alpha \beta)
$$

Hence

$$
P_{V}(\alpha, \beta)=P_{V}(\alpha)
$$

if and only if $\beta$ alin $\alpha$, and $P_{V}(\alpha) \subsetneq_{+} P_{V}(\alpha, \beta)$ if and only if $\beta$ does not almost include $\alpha$. Similarly for $P_{E}(\alpha)$ and $P_{E}(\alpha, \beta)$ under the restriction $\alpha, \beta \in E$.

Definition. $\alpha \operatorname{alin}^{-1} \beta=\beta$ alin $\alpha$.

DEFINITION. Alin ${ }^{-1} S$ is the class of all sets which are almost included in a set of $S$.

\section{Clearly}

$$
\begin{aligned}
& \text { Alin }{ }^{-1} S=\left(\operatorname{Alin} S^{\prime}\right)^{\prime}, \text { Alin } S=\left(\operatorname{Alin}^{-1} S^{\prime}\right)^{\prime}, \\
& Q_{V}(\alpha)=\operatorname{Alin}^{-1} \alpha, Q_{V}(\alpha)=\left(P_{V}\left(\alpha^{\prime}\right)\right)^{\prime}, P_{V}(\alpha)=\left(Q_{V}\left(\alpha^{\prime}\right)\right)^{\prime} .
\end{aligned}
$$

By means of these relations the theory of simple extensions of a dual ideal in a Boolean algebra can easily be dualized to a theory of simple extensions of an ideal in a Boolean algebra. If, for example, $l$ is an ideal in $B$ and $\alpha_{0}, \ldots, \alpha_{n} \in B$, 
then

$$
I_{B}\left(\alpha_{0}, \ldots, \alpha_{n}\right)=I_{B}\left(\alpha_{0}+\cdots+\alpha_{n}\right)
$$

From the fact that $\alpha \equiv \beta$ if and only if $\alpha$ alin $\beta$ and $\beta$ alin $\alpha$ follows:

$$
\langle\alpha\rangle=P_{V}(\alpha) \cdot \xi_{V}(\alpha)
$$

5. We shall now discuss some theorems dealing with the relation between simple extensions of $P$ in $E$ or $V$ and r.e. classes.

DEFINITION. $L(\alpha)$ is the class of all sets which include $\alpha$ (note that $L(\alpha)$ is a dual ideal for every $\alpha$ ). The dual ideal $l$ in $E$ (or $V$ ) is called principal if there exists an $\alpha \in E$ (resp. $\alpha \in V$ ) such that $I=E \cdot L(\alpha)$ (resp. $l=L\left(\alpha_{0}\right)$ ); $\alpha$ is called the generator of $l$.

The principal dual ideals in $V$ can be classified according to the nature of their generators; we shall therefore discuss a classification of sets in $V$ which is relevant to the character of the class $L(\alpha) \cdot F$.

DEFINITION. $\beta$ is a superset of $\alpha$ if $\alpha$ is a subset of $\beta$.

DEFINITION. $\alpha$ is called immune if it is infinite and has no subset in $F-Q ; \alpha$ is called contraimmune if $\alpha^{\prime}$ is infinite and $\alpha$ has no superset in $F-P$.

DEFINITION. $\alpha$ is called normal if it is r.e.; $\alpha$ is called subnormal if it is not r.e. but has a superset in $F-P$.

Every set clearly belongs to exactly one of the three categories: normal, subnormal, contraimmune. Obviously $\alpha \notin R$ whenever $\alpha$ is immune or contraimmune.

We shall use the letter $c$ to denote the cardinal number of the continuum.

T 3.2. 1) There exist exactly c immune sets.

2) There exist exactly denumerably many normal sets, c subnormal sets, and c contraimmune sets.

Proof. To prove that there are $c$ sets of a certain kind it suffices to show that there are at least $c$ sets of that kind, since there are only $c$ sets in $V$.

1) The existence of an immune set follows from the application of $\mathrm{T} 1.2$ to 
the class $F-Q$. An immune set clearly has $c$ infinite subsets, each of which is again immune. Thus there are at least $c$ immune sets.

2 ) There exist exactly denumerably many normal sets, since $F$ is denumerable. When we apply $\mathrm{T} 1.2$ to the class $F^{\prime}-Q$ we see that there exists a set $\gamma \notin R$ such that $\gamma^{\prime}$ does not include any set of $F^{\prime}-(i ;$ thus $\gamma$ is not included in any set of $F-P$, i.e., $\gamma$ is contraimmune. Since $\gamma^{\prime}$ is infinite, there exist disjoint infinite sets $\rho_{1}$ and $\rho_{2}$ such that $\gamma^{\prime}=\rho_{1}+\rho_{2}$. If $\tau \subset \rho_{1}$, then $\gamma+\tau \notin R$, and $\gamma+\tau$ has no superset in $F-P$; it follows that $\gamma+\tau$ is contraimmune. There exist at least $c$ contraimmune sets because $\tau$ can be chosen in $c$ different ways, while different choices of $\tau$ yield different sets $\gamma+\tau$. To prove that there exist exactly $c$ subnormal sets, let $\alpha$ and $\beta$ be two sets in $F-R$ such that $\alpha \subset \beta$ and $\beta-\alpha \notin Q$. None of the $c$ sets $\gamma$ such that $\alpha \subset \gamma \subset \beta$ is contraimmune, as each is included in $\beta$; but only denumerably many of these sets $\gamma$ can be normal. We conclude that there exist at least $c$ subnormal sets.

DEFINITION. The principal dual ideal $L(\alpha)$ is called normal (subnormal, contraimmune) if $\alpha$ is normal (resp. subnormal, contraimmune).

Every principal dual ideal belongs to exactly one of these three categories because it uniquely determines its generator. Every set which is congruent to a normal (subnormal, contraimmune) set is also normal (resp. subnormal, contraimmune), since two congruent sets differ in at most finitely many elements. It follows that all generators of some simple extension of $P$ in $V$ belong to exactly one of the three categories: normal, subnormal, contraimmune.

DEFINITION. A simple extension of $P$ in $V$ is called normal (subnormal, contraimmune) if its generators are normal (resp. subnormal, contraimmune).

T 3.3. 1) Every principal dual ideal in $E$ is r.e. Every normal principal dual ideal in $V$ intersects $F$ in a r.e. class.

2) Every simple extension of $P$ in $E$ is r.e. Every normal simple extension of $P$ in $V$ intersects $F$ in a r.e. class.

Proof. The proofs of the two statements dealing with $E$ are similar to the proofs of the two statements dealing with $F$; we therefore restrict our attention to the latter.

1) We assume $\alpha \neq 0$, the theorem being trivial in case $\alpha=0$. Let $f(m, n)$ be a recursive enumeration of $F-[0]$, and $a(n)$ a recursive function ranging over $\alpha_{\text {; }}$ suppose $\alpha_{m}$ is the range of $f(m, n)$, when we consider $f(m, n)$ as a 
function of $n$. We can now effectively find from $f(m, n)$ and $a(n)$ a recursive function $g_{m}(n)$ ranging over $\alpha+\alpha_{m}$. Then

$$
L(\alpha) \cdot F=\text { Enum } g_{m}(n) \text {, }
$$

and $L(\alpha) \cdot F$ is r.e.

2) We have

$$
P_{V}(\alpha)=\sum_{n=0}^{n=\infty}(\alpha-\nu(n))
$$

and therefore

$$
P_{V}(a) \cdot F=\sum_{n=0}^{n=\infty} L(\alpha-\nu(n)) \cdot F \text {. }
$$

But $L(\alpha-\nu(n)) \cdot F$ is r.e. for every $n$ by 1$)$ and the fact that $\alpha-\nu(n)$ is normal for every $n$. Thus $P_{V}(\alpha) \cdot F$ is r.e. by T 2.1.5.

T 3.4. If $a \notin R$, then:

1) $L(\alpha) \cdot F \underset{+}{\subset} P \leftrightarrow \alpha$ is contraimmune;

2) $P_{V}(\alpha) \cdot F=P \leftrightarrow \alpha$ is contraimmune.

Proof. 1) $L\left(c^{\prime}\right) \cdot F \subsetneq_{+} P \rightarrow \alpha$ has no superset in $F-P \rightarrow a$ is contraimmune. Now suppose $\alpha^{+}$is contraimmune. Then $L(\alpha) \cdot F \subset P$; but if $a \in \alpha$, $\epsilon-\{a\}$ is a set of $P$ which does not include $\alpha$; hence $L(\alpha) \cdot F \subset_{+} P$.

2) Sufficiency. Suppose $I=P_{V}(\alpha)$, where $\alpha$ is contraimmune. Then I. $F \subset P$, by

$$
I \cdot F=\sum_{n=0}^{n=\infty} L(\alpha-\nu(n)) \cdot F
$$

and 1). Let $\pi \in P$ and suppose $m$ is the maximum of $\pi^{\prime}$. Then

$$
\pi \in L(\alpha-\nu(m)) \cdot F \text {. }
$$

Thus $I \cdot F=P$. Necessity. Let $I=P_{V}(\alpha)$ and suppose $I \cdot F=P$. Clearly $L(\alpha) \cdot F \subsetneq_{+} P$ because $L(\alpha) \cdot F \subsetneq_{+} I \cdot F$. Then $\alpha$ is contraimmune by 1$)$.

Our discussion of the nature of the class $P_{V}(\alpha) \cdot F$ is incomplete, since we have considered only the case that $\alpha$ is normal or contraimmune, but not the case that $\alpha$ is subnormal. 
6. Definition. Let $S \neq O$. Every set $\gamma$ such that $S \subset$ Alin $\gamma$ is called a kernel set of $S$. The class of all kernel sets of $S$ will be denoted by Ker $S$.

Using the properties of the alin relation it is easy to show that $\operatorname{Ker} S$ is an extension of $Q$ in $V$ for every nonempty class $S$.

$\mathrm{T}$ 3.5. 1) If $O \subset_{+} S \subset T \subset V-Q$, where $S$ is at most denumerable and $T a$ product system, then Ker $S$ contains a set of $V-R$.

2) If, moreover, $S$ is r.e. we can effectively find a set $\delta \in D$. Ker $S$.

Proof. 1) Since $S$ is nonempty and at most denumerable, we can order the sets of $S$ in an infinite sequence $\alpha_{0}, \alpha_{1}, \ldots$. Let $\beta_{n}$ be the product of the sets $\alpha_{0}, \ldots, \alpha_{n}$ in this sequence; then $\beta_{n} \in V-Q$ for every $n$, since $\beta_{n} \in T$ for every $n$ and $T \subset V-Q$. There exists an enumeration without repetitions of $\beta_{n}$ for every $n_{\text {. Let }} b_{k_{0}}, b_{k_{1}}, \cdots$ be such an enumeration of $\beta_{k}$. Suppose

$$
\begin{aligned}
& c_{0} \underset{d f}{=} b_{00}, \\
& c_{1} \underset{d f}{=} \text { first element in }\left\{b_{1 n}\right\} \text { which is greater than } c_{0}, \\
& c_{2} \underset{d f}{=} \text { first element in }\left\{b_{2 n}\right\} \text { which is greater than } c_{1} \text {, etc. }
\end{aligned}
$$

Let

$$
\gamma \underset{d f}{=}\left\{c_{0}, c_{1}, \ldots\right\}
$$

then $\gamma \in V-Q$. We shall show that $\alpha_{n}$ alin $\gamma$ for every $n$. Clearly $c_{n+k} \in \beta_{n}$ for $k>0$, since $c_{n+k} \in \beta_{n+k} \subset \beta_{n}$. Consequently $\left\{c_{n}, c_{n+1}, \ldots\right\} \subset \beta_{n}$, hence $\beta_{n}$ alin $\gamma$. Then $\alpha_{n}$ alin $\gamma$ as $\alpha_{n} \supset \beta_{n}$. We know that $\gamma \in V-Q$. Let

$$
\gamma_{0}=\left\{c_{0}, c_{2}, c_{4}, \cdots\right\}
$$

then $S \subset P_{V}\left(\gamma_{0}\right)$ and $\gamma_{0} \in V-R$.

2 ) Let $s(n, x)$ be a recursive enumeration of $S$, and $\alpha_{n}$ the range of $s(n, x)$ when we consider $s(n, x)$ as a function of $x$. Then we can effectively find a recursive enumeration without repetitions of the set $\beta_{n}$ mentioned in 1). Thus we can effectively find $c_{n}$ and

$$
\gamma_{0} \in(V-R) \cdot E=D
$$

CoROLLARY. If $I$ is a proper dual ideal in $V$ not containing any finite 
set, then $I \cdot F$ is included in a simple extension of $P$ in $V . I f$, moreover, $I \cdot F$ is r.e., $I$. F is included in a normal simple extension of $P$ in $V$ with a generator in $D$.

Proof. Take $S=T=I \cdot F$ and observe that $O \subset_{+} I \cdot F \subset V-Q$, where $I \cdot F$ is a product system.

We can now give a second proof of part b) of $\mathrm{T} 2.5$, i.e., of the statement: if $M$ is a maximal extension of $P$ in $E$ (or $V$ ), then $M$ (resp. $M \cdot F$ ) is not r.e. For if $M$ were a maximal extension of $P$ in $V$ such that $M \cdot F$ is r.e., there would exist a set $\delta \in D$ such that $M \cdot F \subset P_{V}(\delta)$ by the corollary. Then

$$
M \cdot E \subset P_{V}(\delta) \cdot E=P_{E}(\delta) \subset E-Q
$$

thus $M \cdot E$ would be equal to the simple extension $P_{E}(\delta)$ of $P$ in $E$ because $M \cdot E$ is a maximal extension in $E$. This would contradict the fact that T 3.1.4 also holds if we replace " $V$ " by " $E$ ". Thus $M \cdot F$ is not r.e. Now suppose $M$ is a maximal extension of $P$ in $E$ which is r.e.; then we could prove by a similar reasoning that $M$ is a simple extension of $P$ in $E$; since this is impossible, $M$ cannot be r.e.

\section{Semisimple extensions of $\mathbf{P}$}

1. We have seen in the previous section that every extension of $P$ in $V$ which is obtained by adjoining a finite number of sets to $P$ is a simple extension of $P$ in $V$. We shall now discuss extensions of $P$ in $V$ which are obtained by adjoining denumerably many sets to $P$.

DEFINITION. The extension $l$ of $P$ in $V$ is called a semisimple extension of $P$ in $V$ if there exists a sequence $\left\{\alpha_{n}\right\}$ of sets in $V$ such that

$$
I=\sum_{n=0}^{n=\infty} P_{V}\left(\alpha_{n}\right) .
$$

Any such sequence $\left\{\alpha_{n}\right\}$ is called a $P$-basis of $I$.

Notation. $I=P_{V}\left\{\alpha_{n}\right\}$.

We do not exclude the case that all elements of the $P$-basis $\left\{\alpha_{n}\right\}$ of $I$ are equal. It follows that every simple extension of $P$ in $V$ is also a semisimple extension of $P$ in $V$. The sequence $\left\{\alpha_{n}\right\}$ is called an $\alpha$-sequence if for every pair $\left(m_{s} n\right)$ of nonnegative integers there exists a $k$ such that $\alpha_{m} \alpha_{n}$ alin $\alpha_{k}$. It is readily proved that $\left\{\alpha_{n}\right\}$ is the $P$-basis of a semisimple extension of $P$ in $V$ if 
and only if it is an $C$-sequence. The following theorem can easily be verified.

T 4.1. If $I=P_{V}\left\{\alpha_{n}\right\}$ then:

1) $I=P$ if and only if $a_{n} \in P$ for all $n$;

$I=V$ if and only if $a_{n} \in Q$ for at least one $n$;

$P \underset{+}{C_{+}} c_{+} V$ if and only if $\alpha_{n} \notin P$ for at least one $n$ and $\alpha_{n} \notin Q$ for all $n$.

2) I is a simple extension of $P$ in $V$ if and only if there exists a $k$ such that $\alpha_{n}$ alin $\alpha_{k}$ for all $n$.

3) $I$ is a maximal extension of $P$ in $V$ if anl only if $I \subsetneq_{+} V$ and for every $\beta \in V$ there either exists an $n$ such that $\beta$ alin $\alpha_{n}$ or an $n$ such that $\beta^{\prime}$ alin $a_{n}$.

If $\alpha_{n} \supset \alpha_{n+1}$ for all $n,\left\{\alpha_{n}\right\}$ is called a descending chain with $\prod_{n=0}^{n=\infty} \alpha_{n}$ as inner limit; if $\alpha_{n} \subset \alpha_{n+1}$ for all $n,\left\{\alpha_{n}\right\}$ is called an ascending chain with $\sum_{n=0}^{n=\infty} \alpha_{n}$ as outer limit. If we replace " $\alpha_{n}$ " by " $S_{n}$ " in these definitions, we obtain definitions of a descending chain of classes and its inner limit, and an ascending chain of classes and its outer limit.

T 4.2. 1) There exists an extension of $P$ in $V$ which is semisimple but not simple.

2) Every semisimple extension $I$ of $P$ in $V$ has a descending $P$-basis $\left\{\alpha_{n}\right\}$ such that $I$ is the outer limit of the ascending chain $\left\{P_{V}\left(\alpha_{n}\right)\right\}$ of simple extensions of $P$ in $V$.

Proof. 1) Let

$$
\alpha_{n}=\left\{2^{n}, 2 \cdot 2^{n}, 3 \cdot 2^{n}, \ldots\right\}
$$

then $\left\{\alpha_{n}\right\}$ is an $\alpha$-sequence, since $\alpha_{m} \alpha_{n}=\alpha_{k}$, where $k=\operatorname{Max}(m, n)$. Let $I=P_{V}\left\{\alpha_{n}\right\}$; then $I$ is a semisimple extension of $P$ in $V$. Now $P \subset_{+} I \subset_{+} V$, since $\alpha_{n} \notin Q$ for all $n$ and $\alpha_{n} \notin P$ for $n \geq 1$. If $I$ were a simple extension of $P$ in $V$ there would exist a nonnegative integer $r$ such that $\alpha_{n}$ alin $\alpha_{r}$ for all $n$, in particular $\alpha_{r+1}$ alin $\alpha_{r}$; i.e., $\alpha_{r}-\alpha_{r+1} \in Q$. This contradicts

$$
\alpha_{r}-\alpha_{r+1}=\left\{2^{r}, 3 \cdot 2^{r}, 5 \cdot 2^{r}, \cdots\right\} \notin \subset Q
$$

$I$ is, therefore, not a simple extension of $P$ in $V$.

2) Let $I=P_{V}\left\{\beta_{n}\right\}$, and let $\alpha_{n}$ be the product of $\beta_{0}, \cdots, \beta_{n}$; then $\left\{\alpha_{n}\right\}$ 
is a descending $P$-basis of $I$ such that $I$ is the outer limit of the ascending chain $\left\{P_{V}\left(\alpha_{n}\right)\right\}$.

It is possible to strengthen the second part of the theorem by replacing the words "a descending $P$-basis $\left\{\alpha_{n}\right\}$ " by "a descending $P$-basis $\left\{\alpha_{n}\right\}$ with inner limit $0 "$. Suppose, namely, that $\left\{\alpha_{n}\right\}$ is a descending $P$-basis of $I$, and $\gamma_{n}=$ $\alpha_{n}-\nu(n)$; then $\left\{\gamma_{n}\right\}$ is a descending $P$-basis of $I$ with inner limit o. Since there exist semisimple extensions $I$ of $P$ in $V$ such that $P \subsetneq_{+} I \subsetneq_{+} V$ we see that in general

$$
P_{V}\left\{\alpha_{n}\right\} \neq P_{V}\left(\prod_{n=0}^{n=\infty} a_{n}\right)
$$

Semisimple extensions of $P$ in $E$ can be defined in an obvious manner. Theorems T 4.1 and T 4.2 and their proofs remain valid if we replace $V$ by $E$. Theorems about semisimple extensions of $P$ in $V$ can be dualized to theorems about semisimple extensions of $Q$ in $V$ by means of the relation

$$
\sum_{n=0}^{n=\infty} Q_{V}\left(\alpha_{n}\right)=\left(\sum_{n=0}^{n=\infty} P_{V}\left(\alpha_{n}^{\prime}\right)\right) .
$$

2. Definition. The dual ideal $I$ in $V$ is called pseudopriicipal (p.pr.) if there exists a sequence $\left\{\alpha_{n}\right\}$ of sets in $V$ such that

$$
I=\sum_{n=0}^{n=\infty} L\left(\alpha_{n}\right)
$$

Each such sequence is called a basis of $I$.

Any principal dual ideal in $V$ is p. pr.; the converse is false, for

$$
P=\sum_{n=0}^{n=\infty} L(\epsilon-\nu(n))
$$

is p.pr. but not principal. A necessary and sufficient condition that the sequence $\left\{u_{n}\right\}$ be a basis of a p.pr. dual ideal $n V$ is that for every pair $(m, n)$ of nonnegative integers there exists a $k$ such that $\alpha_{m} \alpha_{n} \supset \alpha_{k}$. We siould distinguish between bases of $I$ and $P$-bases of $I$, in case $I$ is both a p.nr. dual ideal in $V$ and a simple extension of $P$ in $V$. Principal and p.pr. dual jdeals 
in $E$ and principal and p.pr. ideals in $V$ and $E$ can be defined in an obvious manner.

DEFINITION. The sequence $\left\{\alpha_{m}\right\}$ of nonempty sets is r.e. if there exists a recursive function $f(m, n)$ such that for every $m, f(m, n)$, considered as a function of $n$, ranges over $\alpha_{m}$.

T 4.3. 1) An extension of $P$ in $E($ or $V)$ is a semisimple extension of $P$ in $E$ (resp. $V$ ) if and only if it is a p.pr. dual ideal in $E$ (resp. V).

2) Let $I$ be a semisimple extension of $P$ in $E$ (or $V$ ). Then every basis of $I$ is a P-basis of I. Moreover I has a r.e. basis if and only if I has a r.e. P-basis.

Proof. We shall restrict our attention to extensions in $P$ in $V$. The proofs for extensions of $P$ in $E$ are similar.

1) Let $\left\{\alpha_{n}\right\}$ be a $P$-basis of the semisimple extension $I$ of $P$ in $V$. Then $I$ is a p.pr. dual ideal in $V$ with $\alpha_{0}-\nu(0), \alpha_{0}-\nu(1), \alpha_{1}-\nu(0), \cdots$ as basis. Conversely, suppose $I$ is an extension of $P$ in $V$ which is a p.pr. dual ideal in $V$ with $\left\{a_{n}\right\}$ as basis. Since Alin $I=I$, we see that

$$
\alpha_{n} \in I \rightarrow P_{V}\left(\alpha_{n}\right)=\operatorname{Alin} \alpha_{n} \subset I
$$

Hence $I$ is a semisimple extension of $P$ in $V$ with $\left\{\alpha_{n}\right\}$ as $P$-basis.

2) Let $I$ be a semisimple extension of $P$ in $V$. Then every basis of $I$ is a $P$-basis of $I$ [see the second part of the proof of 1$)]$. It follows that a r.e. basis of $I$ is also a r.e. $P$-basis of $I$. If $I$ has a r.e. $P$-basis, say $\left\{\alpha_{n}\right\}, I$ has also a r.e. basis, namely $\alpha_{0}-\nu(0), \alpha_{0}-\nu(1), \alpha_{1}-\nu(0), \cdots$.

T 4.4. 1) A maximal extension of $P$ in $V$ cannot be a p.pr. dual ideal in $V$. 2) A maximal extension of $P$ in $E$ cannot be a p.pr. dual ideal in $E$ with a r.e. basis.

Proof. 1) Suppose $\left\{\alpha_{n}\right\}$ is a basis of the p.pr. dual ideal $I$ in $V$. If $\alpha_{n} \in Q$ for some $n, l$ cannot be a maximal extension of $P$ in $V$; we therefore assume that $\alpha_{n} \notin Q$ for all $n$. The class $S$ over which $\alpha_{n}$ ranges, if $n$ ranges over $\epsilon$, is at most denunierable, and consists of infinite sets. It follows by $\mathrm{T} 1.2$ that there exists a set $y$ such that neither $\gamma$ nor $\gamma^{\prime}$ belongs to $I$; thus $I$ is not maximal.

2) Supr ose $\left\{\alpha_{v_{n}}\right\}$ is a r.e. basis in $E$ of the p.pr. dual ideal $I$ in $E$. We 
can again restrict our attention to the case $\alpha_{n} \notin Q$ for all $n$. The sequence $\left\{\alpha_{n}\right\}$ recursively enumerates a nonempty subclass of $E-Q$; it follows by $\mathrm{T} 2.3$ that there exists a set $\eta \in D$ such that neither $\eta$ nor $\eta^{\prime}$ includes any set of the sequence $\left\{\alpha_{n}\right\}$; thus $I$ is not maximal.

We shall now discuss the question whether maximal extensions of $P$ in $E$ ( or $V$ ) can be semisimple, and if so, whether they can have a r.e. basis in $E$ $(\operatorname{resp} . F)$.

T 4.5. 1) Every extension (in particular every maximal extension) of $P$ in $E$ is a semisimple extension of $P$ in $E$.

2) A maximal extension of $P$ in $E$ cannot have a r.e. basis in $E$.

$3)$ There exist semisimple extensions of $P$ in $E$ which are neither simple nor maximal.

4) No maximal extension of $P$ in $V$ is a semisimple extension of $P$ in $V$.

Proof. 1) Let $I$ be an extension of $P$ in $E$; then $I$ is denumerable, since $P$ and $E$ are denumerable. Let

$$
I=\left[\alpha_{0}, \alpha_{1}, \ldots\right]
$$

then

$$
I=\sum_{n=0}^{n=\infty} L\left(\alpha_{n}\right) \cdot E .
$$

Hence $l$ is a p. pr. dual ideal in $E$ and a semisimple extension of $P$ in $E$.

2) Suppose $M$ is a maximal extension of $P$ in $E$. Then $M$ is both a p.pr. dual ideal and a semisimple extension of $P$ in $E$. If $M$ would have a r.e. $P$-basis, it would be a p.pr. dual ideal in $E$ with a r.e. basis, by T 4.3 .2 ; this is, however, impossible in view of $\mathrm{T} 4.4 .2$.

3) I et $I=P_{E}\left\{\alpha_{n}\right\}$, where $\left\{\alpha_{n}\right\}$ is the sequence of sets used in the proof of $\mathrm{T}$ 4.2.1. Then we can easily show that $P \subset_{+} I \subset_{+} E$ and that $I$ is not a simple extension of $P$ in $E$. Now' $I$ has a r.e. basis, because it has the r.e. $P$-basis $\left\{\alpha_{n}\right\}$; thus $I$ is not a maximal extension of $P$ in $V$.

4) Every semisimple extension of $P$ in $V$ is a p.pr. dual ideal in $V$ by T 4.3.1. But a MDI in $V$ cannot be p.pr., by $\mathrm{T} 4.4 .1$. 
We see, therefore, that the relation between semisimple extensions of $P$ and maximal extensions of $P$ is very different in $E$ and $V$. This is obviously related to the fact that $E$ is denumerable and $V$ nondenumerable.

3. While we have been able to solve the problem "Which semisimple extensions of $P$ intersect $F$ in a r.e. class?" for the Boolean algebra $E$, we have solved only some special cases of this problem for the Boolean algebra $V$.

T 4.6. 1) A dual ideal in $E$ is r.e. if and only if it has a r.e. basis in $E$.

2) A semisimple extension of $P$ in $E$ is r.e. if and only if it has a r.e. P-basis in E.

Proof. 1) Suppose $\left\{\alpha_{n}\right\}$ is a r.e. basis in $E$ of the p.pr. dual ideal $I$ in $I$. Then

$$
I=\sum_{n=0}^{n=\infty} L\left(a_{n}\right) \cdot E
$$

hence $l$ is r.e., by $\mathrm{T} 2.5$ and $\mathrm{T} 3.3 .1$. If the dual ideal $l$ in $E$ is r.e., its elements can be written in a r.e. sequence $\left\{\alpha_{n}\right\}$; this r.e. sequence $\left\{\alpha_{n}\right\}$ is one of the bases of $l$.

2) This follows from 1 ) and $T 4.3$.

COROLLARY. There exist semisimnle extensions of $P$ in $E$ which are not r.e.

Proof. A maximal exten or of $P$ in $E$ is semisimple; it is not r.e., by $\mathrm{T}$ 4.6.1 and $\mathrm{T}$ 4.5.2.

Definition. A p.pr. dual ideal in $V$ is called normal (subnormal, contraimmune), if it has a basis consisting entirely of normal (resp. subnormal, contraimmune) sets.

Definition. A semisimple extension of $P$ in $V$ is called normal (subnormal, contraimmune), if it has a $P$-basis consisting entirely of $r$ rmal ( 1 - $s$. subnormal, contraimmune) sets.

It is easily verified that a semisimple extensic $r$ oi $P$ in $V$ is a normal (subnormal, contraimmune) semisimple extension of ' in $V$ if and only if it is a normal (resp. subnormal, contraimmune) p.pr. dual ideal in $V$. 
T 4.7. 1) A normal p.pr. dual ideal (or semisimple extension of $P$ ) in $V$ intersects $F$ in a r.e. class if and only if it has a r.e. basis (resp. r.e. P-basis) in $F$.

2) A contraimmune semisimple extension of $P$ in $V$ intersects $F$ in $P$.

Proof. 1) The proofs are sinilar to those of the two parts of T 4.6.

2) This follows from T 3.1.2.

4. 1 4.8. Suppose I does not contain any finite set.

1) Every dual ideal I in $E$ (or every p.pr. dual iáeal I in V) is included in a proper simple extension of $P$ in $V$.

2) Every dual ideal I in $t$ with a r.e. basis in E (or every p.pr. dual ideal I in $V$ with a r.e. basis in $F$ ) is included in a simple extension of $P$ in $E$ (resp. $V$ ) with a generator in $D$.

Proof. 1) By applying the diagonal procedure used in the proof of $\mathrm{T} 3.5$ to a basis $\left\{\alpha_{n}\right\}$ of $I$ we can prove the existence of a set $\gamma \in V-R$ such that $I \subset P_{V}(\gamma)$.

2) If $\left\{\alpha_{n}\right\}$ is r.e., the diagonal procedure becomes effective and $\gamma \in D$.

COROLLARY. 1) Every proper semisimple extension of $P$ in $V$ is included in a proper simple extension of $P$ in $V$. 2) Every proper semisimple extension of $P$ in $E$ (or $V$ ) with a r.e. basis in $E$ (resp. $F$ ) is included in a simple extension of $P$ in $E$ (resp. $V)$ with a generator in $U$.

5. We refer to the introduction for the definition of a dual ideal in $E$ (or $V$ ) which is constructive in the second sense.

REMARK. A dual ideal in $E$ is constructive in the second sense if and only if it has a r.e. basis in E. A dual ideal in $V$ is constructive in the second sense if and only if it is a p.pr. dual ideal in $V$ with a r.e. basis in F.

T 4.9. A MDI in $E$ (or $V)$ is constructive in the second sense if and only if it is atomic.

Proof. We shall denote the class of all sets which include a set of $S$ by $\bar{S}$.

a) Let $M$ be an atomic MDI in $V$, say $\|=L(k)$. Then $M=\bar{S}$, where $S=[\{k\}]$; moreover, $S$ is r.e., since $S=\operatorname{Enum} f(m, n)$, where $f(m, n)=k$ for every $m$ and 
$n$. The proof that an atomic MDI in $E$ is constructive in the second sense can be obtained by replacing " $V$ ", " $L(k)$ ", " "S", respectively by " $E$ ", " $L(k) \cdot E$ ", "S $\cdot E "$.

b) Let $M$ be a maximal extension of $P$ in $V$. Then $M$ is not a p.pr. dual ideal in $V$, by T 4.4.1; thus $M$ is not constructive in the second sense. If, however, $M$ is a maximal extension of $P$ in $E, M$ is a p.pr. dual ideal in $E$, by 7 4.5.1; but $M$ has no r.e. basis in $E$ by T 4.5 .2 ; thus $M$ is not constructive in the second sense.

6. We have seen that a MDI in $E$ ( or $V$ ) is constructive if and only if it is atomic according to each of the two definitions for a constructive dual ideal which we discussed. The question now arises whether these two definitions are equivalent.

T 4.10. 1) The two definitions are equivalent in the Boolean algebra E.

2) The two definitions are equivalent for p.pr. dual ideals in the Boolean algebra $V$.

3 ) Every dual ideal in $V$ which is constructive in the second sense is also constructive in the first sense; the converse however, is false.

Proof. 1) All dual ideals in $E$ are p.pr., since $E$ is denumerable. A p.pr. dual ideal in $E$ is constructive in the first sense if and only if it has a r.e. basis in $E$, by $\mathrm{T} 4.6 .1$; and a p. pr. dual ideal in $E$ is constructive in the second sense if and only if it has a r.e. basis in $E$.

2) The only dual ideals in $V^{\prime}$ which can be constructive in the second sense are the normal p.pr. ones; and for these the two definitions agree, by $\mathrm{T}$ 4.7.1.

3) If a dual ideal in $V$ is constructive in the second sense, it is p.pr.; hence it is also constructive in the first sense, by part 2). Let $I$ be a contraimmune semisimple extension of $P$ in $V$. Then $I \cdot F=P$, where $P$ is r.e.; hence $I$ is constructive in the first sense. To prove that $I$ is not constructive in the second sense it suffices to show that $I$ is not a normal semisimple extension of $P$ in $V$. Suppose $I=P_{V}\left\{\alpha_{n}\right\}$, where $\alpha_{n} \in F$ for every $n$; then $\alpha_{n} \in P$ for every $n$, since $I \cdot F=P$. Consequently $I=P$; this however, is false, for the fact that $I$ contains a contraimmune set implies $P \subsetneq_{+} I$. The assumption that $I$ is a normal semisimple extension of $P$ in $V$ leads, therefore, to a contradiction. 


\section{REFERENCES}

1. O. Frink, Representations of Boolean algebras, Bull. Amer. Math. Soc. 47 (1941), $755-756$.

2. S. C. Kleene, Recursive predicates and quantifiers, Trans. Amer. Math. Soc. 53 (1943), $41-73$.

3. F. L. Post, Recursively enumerable sets of positive integers and their decision problems, Bull. Amer. Math. Soc. 50) (1944), 284-316.

4. P. C. Rosenbloom, The elements of mathematical logic, New York, 1950.

5. W. Sierpinski, Sur les ensembles presque contenus les uns dans les autres, Fund. Nath. 35 (1948), $141-150$.

6. M. H. Stone, The theory of representations for Boolean algebras, Trans. Amer. Soc. 1) (1936), 37-111.

7. A. Tarski, Une contribution '̀ la théorie de la mesure, Fund. Nath. 15 (1930), $42-50$.

8. - Ideale in vollständigen Mengenkörpern I, Fund. Math. 32 (1939), 45-63.

\section{SYRACUSE UNIVERSITY}





\section{PACIFIC JOURNAL OF MATHEMATICS}

\section{EDITORS}

\author{
R. M. Foeinson \\ University of California \\ Berkeley 4, California \\ E. HEWITt \\ University of Washington \\ Seattle 5, Washington
}

R. P. DILWOR TH

California Institute of Technology

Pasadena 4, California

E. F. BECKENBACH

University of California

Los Angeles 24, California

\section{ASSOCIATE EDITORS}

$\begin{array}{llll}\text { H. BUSEMANN } & \text { P. R. HALMOS } & \text { BøRGE JESSEN } & \text { J. J. STOKER } \\ \text { HERBERT FFDERER } & \text { HEINZ HOPF } & \text { PAUL LÉVY } & \text { E. G. STR AUS } \\ \text { MARSHALL IIALI } & \text { R. D. JAMES } & \text { GEORGE PÓLYA } & \text { KỎSAKU YOSIDA }\end{array}$

\section{SPONSORS}

UNIVERSITY OF BRITISH COLUMBIA CAIIFORNIA INSTITUTE OF TECHNOLOGY UNIVERSITY OF CALIFORNIA, BERKELEY UNIVERSITY OF CAILIFORNIA, DAVIS UNIVERSITY OF CALIFORNIA, LOS ANGELES UNIVERSITY OF CALIFORNIA, SANTA BARBARA UNIVERSITY OF NEVADA OREGON STATE COLLEGE UNIVERSITY OF OREGON
UNIVERSITY OF SOUTHERN CALIFORNIA STANFORD RESEARCH INSTITUTE STANFORD UNIVERSITY WASHINGTON STATE COLLEGE UNIVERSITY OF WASHINGTON

AMERICAN MATHEMATICAL SOCIETY NATIONAL BUREAU OF STANDARDS, INSTITUTE FOR NUMERICAL ANALYSIS

$$
\begin{gathered}
\text { Vari-Type Composition by } \\
\text { Elaine Barth } \\
\text { Delores Wierman } \\
\text { With the cooperation of } \\
\text { E. F. Beckenbach } \\
\text { E. G. Straus }
\end{gathered}
$$

Printed in the United States of America by Edwards Brothers, Inc., Ann Arbor, Michigan 


\section{Pacific Journal of Mathematics}

\section{Vol. 3, No. 1 \\ March, 1953}

Herbert Busemann, Volume in terms of concurrent cross-sections ......... 1

L. Carlitz, Some special equations in a finite field ................. 13

Homer V. Craig and Billie Braden Townsend, On certain metric

extensors ....................................... 25

Philip J. Davis and Henry Pollak, Linear functionals and analytic

continuation problems ............................. 47

Jacob C. E. Dekker, The constructivity of maximal dual ideals in certain

Boolean algebras ................................. 73

Harley M. Flanders, The norm function of an algebraic field extension .... 103

Marshall Hall, Subgroups of free products . . . . . . . . . . . . . . . . . 115

Israel (Yitzchak) Nathan Herstein, Finite multiplicative subgroups in

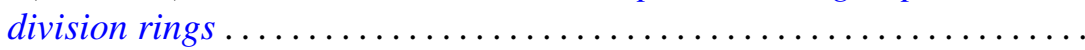

Joseph Lawson Hodges, Jr. and Murray Rosenblatt, Recurrence-time moments in random walks ............................ 127

Alfred Horn, The normal completion of a subset of a complete lattice and lattices of continuous functions ........................ 137

Fulton Koehler, Estimates for the errors in the Rayleigh-Ritz method...... 153

M. H. Martin, The Monge-Ampère partial differential equation

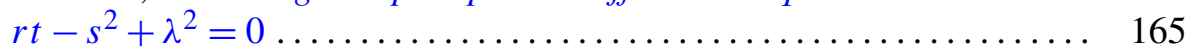

John E. Maxfield, Normal k-tuples ......................... 189

Jack E. McLaughlin, Structured theorems for relatively complemented lattices............................................ 197

William H. Mills, A system of quadratic Diophantine equations ......... 209

T. S. Motzkin, Ernst Gabor Straus and F. A. Valentine, The number of farthest points ................................. 221

G. Power, Forces on the boundary of a dielectric ............... 233

Ralph Gordon Selfridge, Approximations with least maximum error....... 247 